\title{
Photoacoustic Microscopy Provides Early Prediction of Tissue Necrosis in Skin Avulsion Injuries
}

\author{
Dong Zhang \\ Ying Yuan \\ Hao Zhang \\ Xinzeyu Yi \\ Weidong Xiao \\ Aixi Yu (D)
}

Department of Orthopedics Trauma and Microsurgery, Zhongnan Hospital of Wuhan University, Wuhan, People's Republic of China
Correspondence: Aixi Yu; Weidong Xiao Department of Orthopedics Trauma and Microsurgery, Zhongnan Hospital of Wuhan University, Wuhan, People's Republic of China Tel +86-027-67813120; +86-|8707|82868

Email yuaixi@whu.edu.cn; xiaoweidong@whu.edu.cn
Background: Skin avulsion injuries caused by high-energy traffic and machinery accidents are important topics in the field of repair and reconstruction. The injury generates a skin flap with uncertain vascular basis resulting in ischemic necrosis of the distal portion of the flap. Yet there is lack of reliable way for estimating the extent of blood supply in damaged tissue, which has limited the possibility of prompt surgical intervention. Recent studies have confirmed that photoacoustic microscopy imaging has a wide range of applications in the biomedical field owing to its good performance in angiography.

Methods: In our study, we successfully surgically induced skin avulsion injury on mice hindlimbs. Then, we used this novel approach to image skin microcirculation and predict skin necrosis with impaired blood supply after injury in live mice.

Results and Conclusion: All skin tissues in the avulsed hindlimb flap group show different levels of necrosis at the end of the observation period. The "dark zone" with impaired microcirculation in PAM images, which continuously extends over time, was seen as a prediction of necrosis of skin tissue and at $60 \mathrm{~min}$ after surgery was similar to the area of clinical necrosis on postoperative day 7. All these indicate that photoacoustic microscopy imaging is a feasible, precise, high-resolution, non-invasive technique for early prediction of necrosis in skin avulsion injury, providing a promising tool for surgeons to manage the injury.

Keywords: skin avulsion injuries, photoacoustic microscope, monitoring microcirculation

\section{Introduction}

The skin avulsion injury also called Morel-Lavallée lesions is a tough surgical challenge in an emergency context due to its high morbidity and mortality. ${ }^{1,2}$ The injury is caused by the vertical shearing force during motor vehicle or machinery accidents, resulting in abrupt detachment of the skin and subcutaneous tissue from the underlying fascia, muscle and periosteum. ${ }^{3}$ It has been classified into four patterns of injury: abrasion/avulsion, non-circumferential degloving, circumferential single plane and circumferential multi-plane degloving. ${ }^{4}$ It is generally acknowledged that microcirculation involves subdermal capillaries which play an indispensable role in the transport of nutrients and oxygen to the cells and maintain the perfusion of different layers of the skin and subcutaneous tissue. ${ }^{5-8}$ So once the microcirculation of skin and subcutaneous tissue is disrupted by the forceful detachment in the skin avulsion injury, skin necrosis of the avulsed area will happen. Sometimes the skin may remain intact after the initial injury with the latent 
harm to blood supply hidden, but delayed necrosis will develop from hours to several weeks after trauma. ${ }^{9}$ Thus, it is imperative to accurately assess the vascular basis of the avulsed skin for early prediction of tissue viability. Currently, the evaluation of the microcirculation of an avulsed flap is mainly assessed by clinical observation including color, skin temperature, dermal bleeding and capillary refill, but these clinical indicators are subjective and have limited sensitivity and/or specificity. ${ }^{10,11}$ In addition, although routine imaging techniques including ultrasound, magnetic resonance imaging, and computed tomography can be used to diagnosis the injury and determine whether or not the injury has underlying hematoma or fracture, their resolution is still unsatisfactory to reveal the cutaneous microcirculation. ${ }^{12-14}$ Due to their certain intrinsic limitations, it is difficult for surgeons to accurately predict the extent of an avulsed flap and decide beforehand what to remove or conserve, which really predisposes the injury to secondary infection and subsequent skin necrosis. Therefore, an available imaging technique which can accurately evaluate the vascular basis of the damaged tissue is urgent.

As a fast emerging, noninvasive biomedical imaging modality based on the effect of photoacoustics, photoacoustic microscope imaging (PAM) is currently capable of bridging this gap with its high-resolution images of blood vessels within biological tissues. ${ }^{15-17}$ In photoacoustic microscope imaging, non-ionizing laser pulses are delivered into biological tissues; the delivered energy will be absorbed and converted into heat, resulting in the generation of ultrasound in the tissue, then the photoinduced ultrasound is detected and converted into electrical signals by ultrasonic transducer, and then the signals are analyzed to reconstruct images. ${ }^{18}$ Studies have demonstrated that optical absorption is closely associated with physiological properties. ${ }^{19}$ Since blood usually has orders of magnitude higher absorption than surrounding tissues, there is sufficient endogenous contrast for PAM to visualize blood vessels. Most importantly, it is characterized by the high resolution, deep penetration, quantitative analysis, real time and noninvasiveness when visualizing the microcirculation of tissue. Recently, surging studies have shown that PAM can be used in vivo for observing the microcirculation in many physiological and pathophysiological conditions, such as subcutaneous microvasculature imaging, brain functional imaging, and tumor angiogenesis/ treatment response monitoring, etc. ${ }^{20}$
Although it has long been realized that photoacoustic microscope imaging has a good ability in observing the microcirculation, there are no studies in which PAM hasbeen involved beforehand for visualizing the microcirculation in a skin avulsion injury. Therefore, the aim of this study was to describe this noninvasive imaging method for predicting viable and nonviable tissues in skin avulsion mouse models by evaluating the cutaneous microcirculation in a very quantitative and precise way.

\section{Methods and Materials}

\section{Photoacoustic Microscopy System}

The schematic of the photoacoustic microscopy imaging system is shown in Figure 1. A miniature laser (532-2-V, Innolas, Germany), which operates a wavelength of 532 $\mathrm{nm}$ with a pulse width of $12 \mathrm{~ns}$ and a repetition rate of 50 $\mathrm{kHz}$, is used as the irradiation source. The laser beam is coupled into a single-mode fiber (460HP, Thorlab, USA) via a fiber collimator (PAF-X-7-A, Thorlab, USA). Then the laser beam from the fiber is collimated and focused by fiber collimator (F240FC-532, Thorlabs, USA) and 4× objective (GCO-2111, Daheng Optics, China) to excite the sample. The energy density on the tissue surface is around $11 \mathrm{~mJ} / \mathrm{cm}^{2}$, which is below the ANSI safety limit of $20 \mathrm{~mJ} / \mathrm{cm}^{2}$. $^{21}$

To realize the aim of maximizing the sensitivity and signal-to-noise ratios, we make ultrasonic detection and optical excitation confocal. The imaging detector consists of two major components: objective lens and ultrasound transducer. The photoacoustic signals from the sample are collected by a self-made ultrasonic transducer $(35 \mathrm{MHz}$, Doppler, China), the diameter of the element is $8 \mathrm{~mm}$ and with a $3 \mathrm{~mm}$ center hole for the exit of the laser beam, then amplified with a $50 \mathrm{~dB}$ low noise amplifier (LNA-650, RF Bay, USA), finally digitized using a dual-channel data acquisition card (M3i.3221, Spectrum, Germany; sampling rate of $250 \mathrm{MHz}$; 14-bit dynamic range).

\section{Establishing an Animal Model}

Our experiments were performed in compliance with the guidelines of the Institutional Animal Care and Use Committee (IACUC) of Wuhan University. The experimental protocol was approved by the Committee on the Ethics of Animal Experiments of Wuhan University, Wuhan, China (Approval number: 2019173). We purchased female BALB/ c mice at age 5-6 weeks and weight 20-30 g from the laboratory Animal Center of Wuhan (China). Animals 


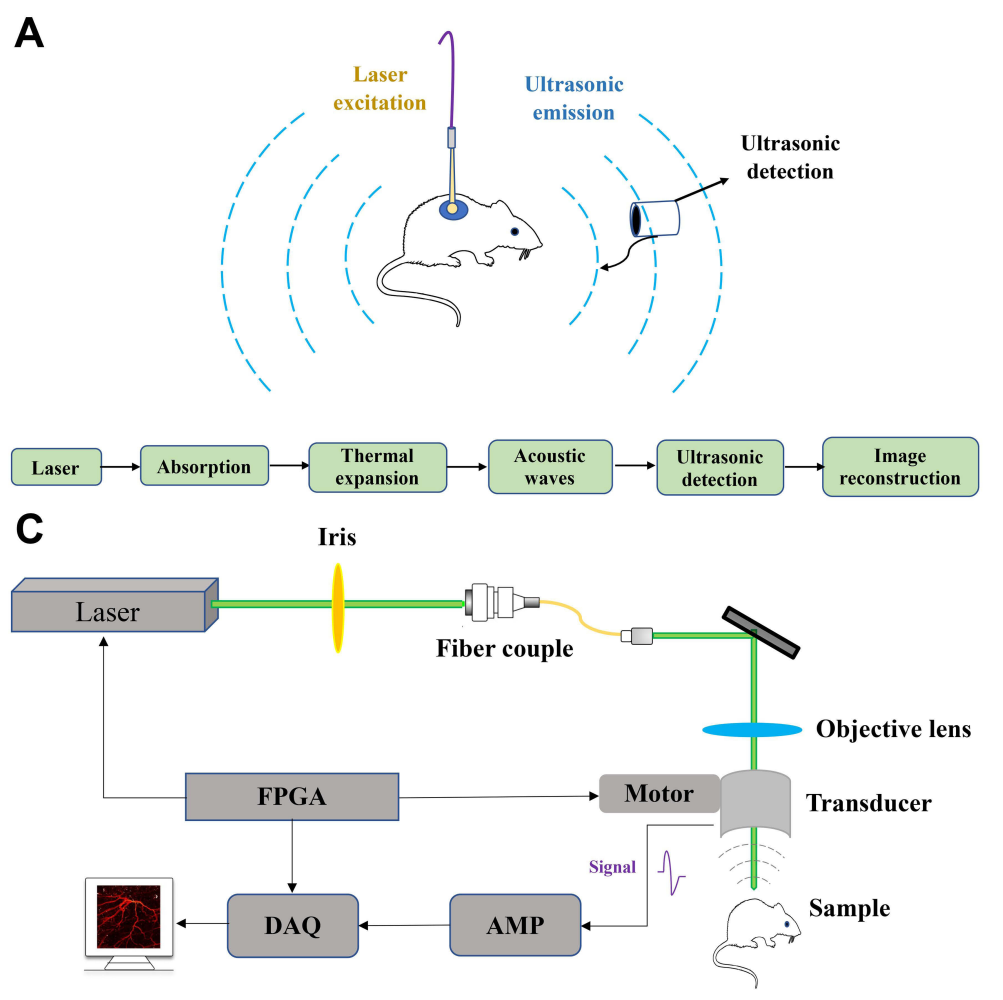

\section{B}

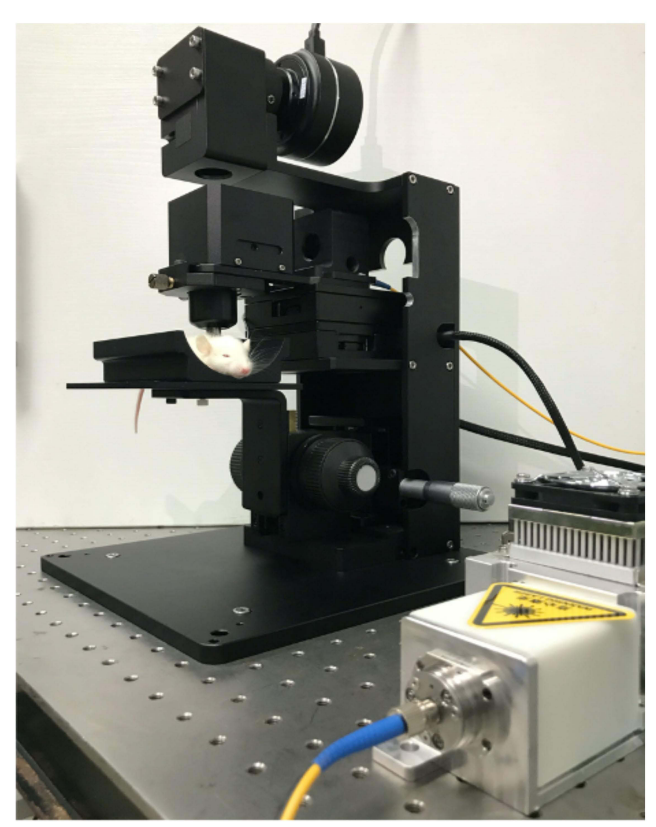

Figure I (A) The mechanism of photoacoustic microscopy, (B and $\mathbf{C}$ ) the system used for in vivo photoacoustic imaging of mouse limb. Abbreviations: FPGA, field programmable gate array; DAS, data acquisition system; Amp, amplifier.

were allowed to acclimate to their holding facility for at least 5 days before the start of the experiment. All surgeries and clinical assessments were performed by a senior surgeon. The experimental procedures, including both operation and measurements, were under general anesthesia with ketamine hydrochloride (30 mg/kg body weight). All efforts were made to minimize animal suffering.

The protocols of establishing skin avulsion model have been described in detail previously. ${ }^{22}$ In our study, 18 mice were used to establish the model, and mice were randomly divided into avulsed hindlimb flap group and sham group. The anesthetized mouse was placed in prone position, upper and lower limbs were stretched slightly and fixed, the lower limbs was shaved and disinfected with $70 \%$ ethyl alcohol. Just like described above, in avulsed hindlimb flap group, a circular incision of skin was made in proximal hindlimb along inguinal and gluteal creases, then the skin was affixed with a surgical towel clamp and was avulsed down to distal femur, finally the avulsed skin was sutured back in its original position using nylon 5-0 suture. In sham group, a circular incision along inguinal and gluteal creases was performed and then sutured in situ.

\section{In vivo Imaging}

Prior to photoacoustic imaging, the hindlimb was shaved and chemically depilated with a depilatory cream, to try to realize the aim of acquiring a high-quality image by making the skin surface evennand the focal plane suitable for the region of interest. Photoacoustic microscopy imaging acquisition happened before surgery; immediately after surgery; 30 minutes and 60 minutes after surgery. The "dark zone" with hardly any intact microvessels was counted as predictive of necrosis. After $12 \mathrm{~h}$ observation, every mouse was raised in a separated cage in an airconditioned room and fed a standard laboratory diet for 7 days. The overall 7-day duration was an adequate amount of time to exhibit the clinical necrosis of avulsion tissue. Digital photographs were acquired before surgery and on postoperative day 7 to demonstrate avulsion flap necrosis. The avulsion flap sample after 7-day observation was collected for standard histological examination by Hematoxylin-Eosin staining (H\&E). In addition, the score of tissue lesions were analyzed via National Pressure Ulcer Advisory Panel (NPUAP) Scale (Table 1). ${ }^{23}$ 
Table I The score of tissue lesions

\begin{tabular}{|l|l|}
\hline Stage & Histological Findings \\
\hline 0 & Normal skin \\
\hline 1 & $\begin{array}{l}\text { Visible inflammation but intact skin with non-blanchable } \\
\text { redness of a localized area }\end{array}$ \\
\hline 2 & $\begin{array}{l}\text { Partial thickness loss of dermis presenting as a shallow open } \\
\text { ulcer }\end{array}$ \\
\hline 3 & $\begin{array}{l}\text { Full thickness tissue loss. Subcutaneous fat may be visible } \\
\text { but bone, tendon or muscle are not exposed }\end{array}$ \\
\hline 4 & $\begin{array}{l}\text { Full thickness tissue loss with exposed bone, tendon or } \\
\text { muscle }\end{array}$ \\
\hline
\end{tabular}

\section{Quantitative Assessment and Statistical}

\section{Analysis}

The original data were processed to generate images using our developed algorithm implemented in LabVIEW (2016, National Instruments, USA), then image post-processing and analysis was managed using MATLAB (R2014b, the MathWorks, USA) and ImageJ (National Institutes of Health, USA). Vascular density and PA intensity in region of interest (ROIs) were computed to quantitatively assess the change of the vascular properties in ROIs. Mice were followed weekly for clinical evaluation. Black color, dehydration, and eschar formation of skin were defined as clinical signs of skin necrosis. The necrosis was predicted on POD 7 digital images by two different surgeons based on the clinical assessment.

\section{Results}

The mechanism of photoacoustic microscopy is shown in Figure 1A. After a specific wavelength laser pulse is delivered into tissue, the energy is partly absorbed by hemoglobin and converted into heat which leads to transient thermoelastic expansion of red blood cells and thus generates the broadband frequency ultrasound named photoacoustic signals. The generated photoacoustic signals are detected and converted into electrical signals via an ultrasonic transducer. Next, electrical signals are obtained by the data acquisition system and finally analyzed to produce vessel images by a computer. The schematic of the photoacoustic microscopy imaging system is shown in Figure 1B and C. Avulsed hindlimb flap following a proximal circumferential incision is shown in Figure 2A, the microvascular networks in hindlimb were visualized via PAM (Figure 2B), and Figure 2C and D show the digital and PAM image of region of interest in hindlimb. In order to verify the ability of PAM in early prediction of tissue necrosis, and to further validate its clinical potential to help in monitoring of skin avulsion injuries, the experiments shown in Figure 2 were performed. After avulsed hindlimb flap group and control group were created, we evaluated preoperative and postoperative PAM images and digital images of ROIs. On the 7th postoperative day, the decision about tissue viability were assessed by three experienced surgeons based on digital photographs and clinical assessment at the end of the observation period. At the start of the observation, ROIs in the two groups were healthy, however, skin tissue necrosis happened at the end of the observation period in the avulsed hindlimb flap group. On the contrary, the necrosis was not seen in the sham group on postoperative day 7 . The H\&E staining and ulcer scores in the two groups in Figure 3 also show this conclusion. All this indicates that our skin avulsion injury model is reliable, and has laid an important foundation for this research. Through the PAM image system, microvascular networks in ROIs could be observed clearly during the entire course of experiment; and in the avulsed hindlimb flap group, all PAM images at $60 \mathrm{~min}$ after operation show a "dark zone" with hardly any intact microvessels, which was counted as predictive of necrosis. As is shown in Figure 4, the representative PAM images of ROIs in the two groups were obtained before the surgery and immediately, at 30 minutes, and 60 minutes after the operation.

To gain quantitative insight into change of microvascular networks, the PA intensity and vascular density were calculated at every specific time point. The analysis in Figures 5 and 6 shows that PA intensity and vascular density decreased significantly versus time when compared with the control group, which shows great potential to help predict the necrosis of skin in avulsed injury.

\section{Discussion}

As an important topic in the field of repair and reconstruction, better assessment of cutaneous microcirculation in skin avulsion injuries may provide important diagnostic information for early prediction of skin necrosis. ${ }^{5,7}$ After the occurrence of this injury, how to non-invasively and accurately assess skin status remains a challenge to the surgeon and the patients. ${ }^{24}$ In general, early and quantitative assessment of microvessels and prompt surgical intervention are thought to be key contributors to deal with this disease before complications arise. ${ }^{25}$ For minor injuries, anticoagulants, $\mathrm{A}-\mathrm{V}$ shunts, etc. can be used to deal with the problem, but debridement and soft tissue reconstruction may be optimal choices for another situations. ${ }^{5,26}$ Therefore, it is very important for treatment option selection to evaluate the extent of the injury based on 

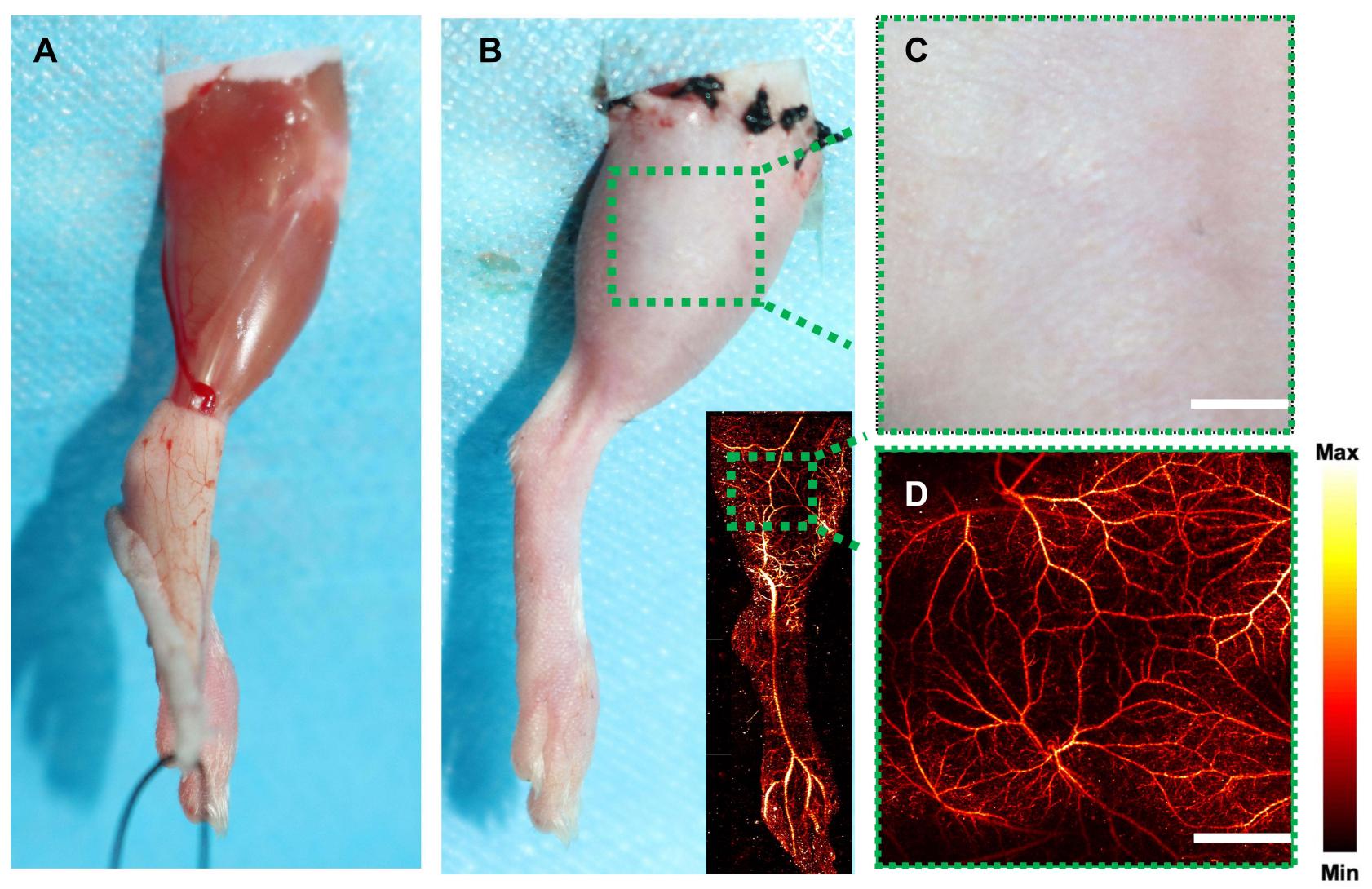

Figure 2 (A) Avulsed hindlimb flap following a proximal circumferential incision. (B-D) The digital and PAM imaging of region of interest in mouse limb.
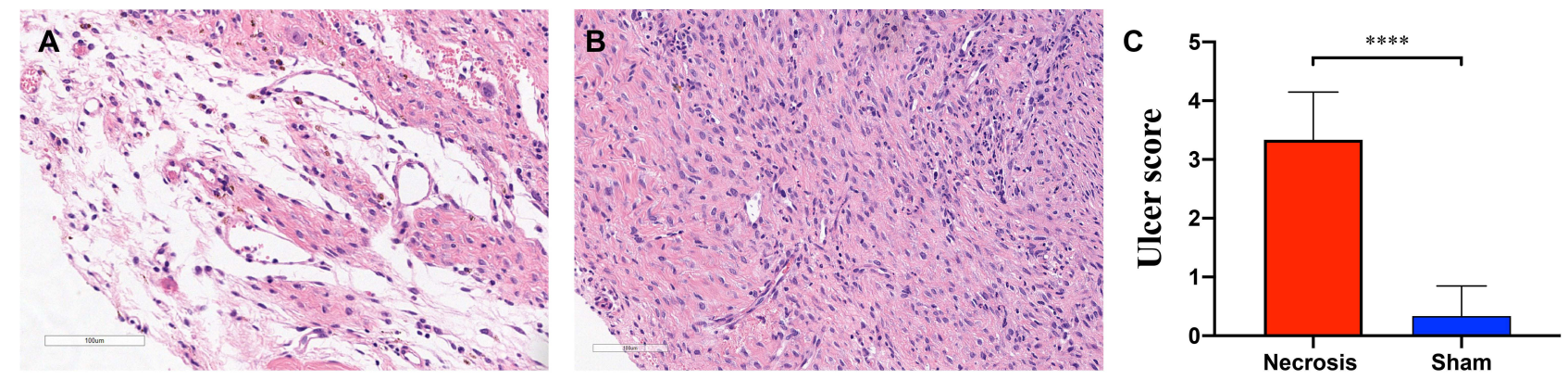

Figure 3 (A and B) H\&E staining in avulsed hindlimb flap group and sham group. (C) The ulcer scars in two groups and the results show that the ulcer scars in avulsed hindlimb flap (necrosis) group were significantly higher than sham group. ${ }^{* * * *} \mathrm{p}<0.000$ I.

the assessment of microvessels. Although the condition has shown the need for a reliable technique reflecting true physiological and pathological behaviors of cutaneous circulation, the effective imaging modalities for prediction of tissue necrosis in skin avulsion injuries remain largely limited. ${ }^{27}$ Currently, the most often used imaging tools for skin avulsion injuries are ultrasound, x-ray angiography, magnetic resonance angiography, and computed tomographic angiography. All of these show various intrinsic limitations; for example, they are not able to obtain images in a noninvasive, dynamic, in vivo and real-time way; unsatisfactory resolution makes it difficult, if not impossible, for them to provide detailed visualization of the cutaneous micro-blood vessel networks; more importantly, x-ray angiography, magnetic resonance angiography, and computed tomographic angiography need to inject angiographic agents which really disturb the intrinsic physiology of the microcirculation and hinder longitudinal monitoring. ${ }^{6,10,28}$ Therefore, a clinical and technical imaging platform that could image true cutaneous microvessels through a noninvasive, precise and quantitative way is in 


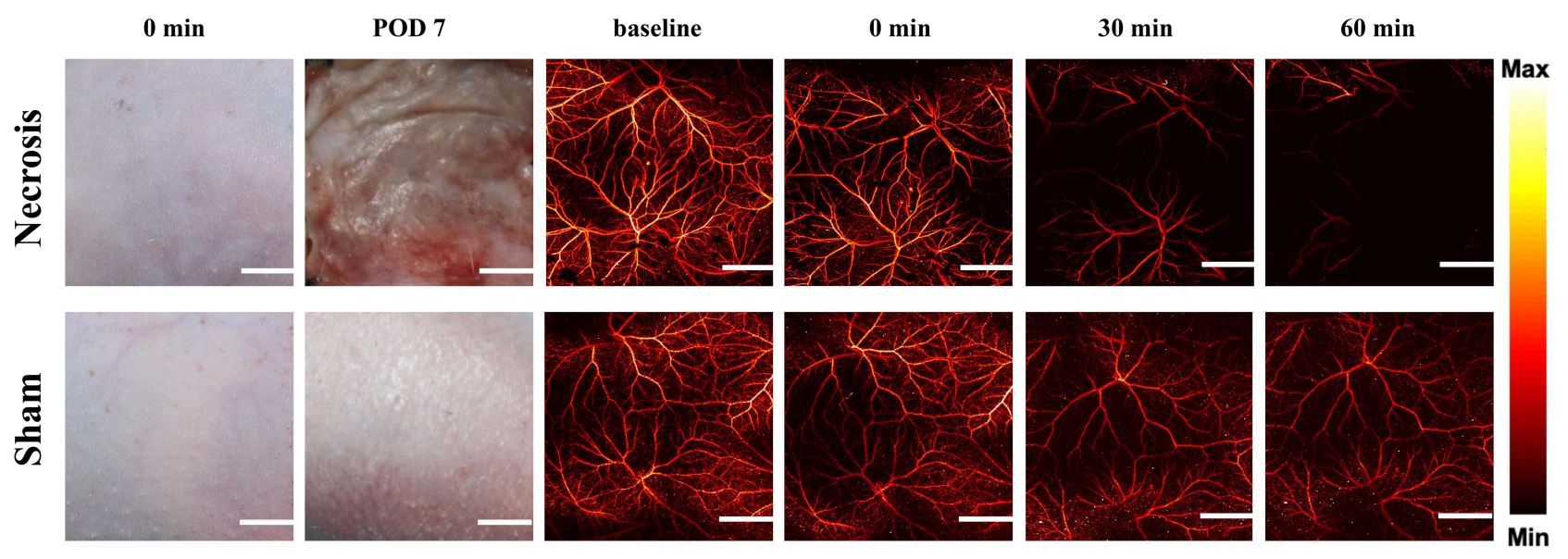

Figure 4 Clinical outcome at POD 7 and PAM imaging at several time points in region of interest between avulsed hindlimb flap group and sham group.

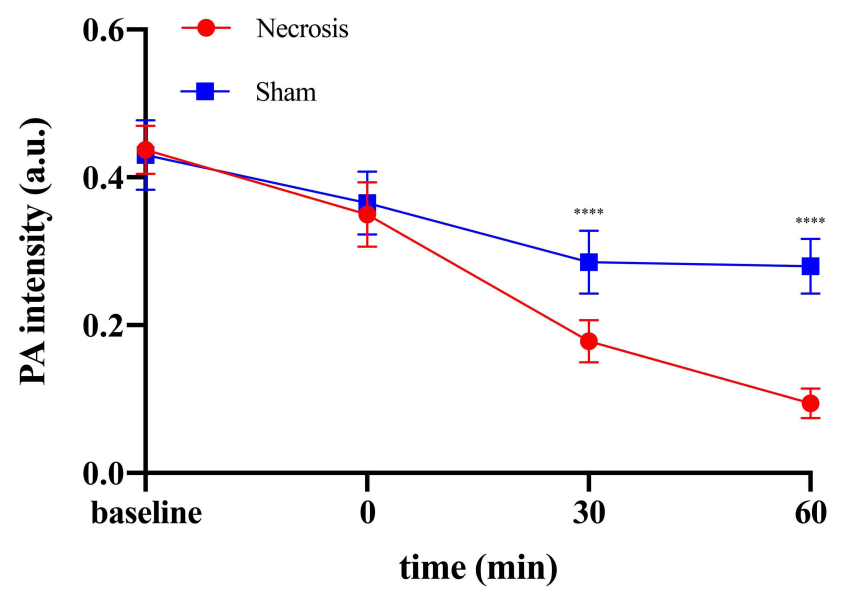

Figure 5 Quantitative analysis of PA intensity in ROls detected by PAM between two groups. $* * * * p<0.0001$.

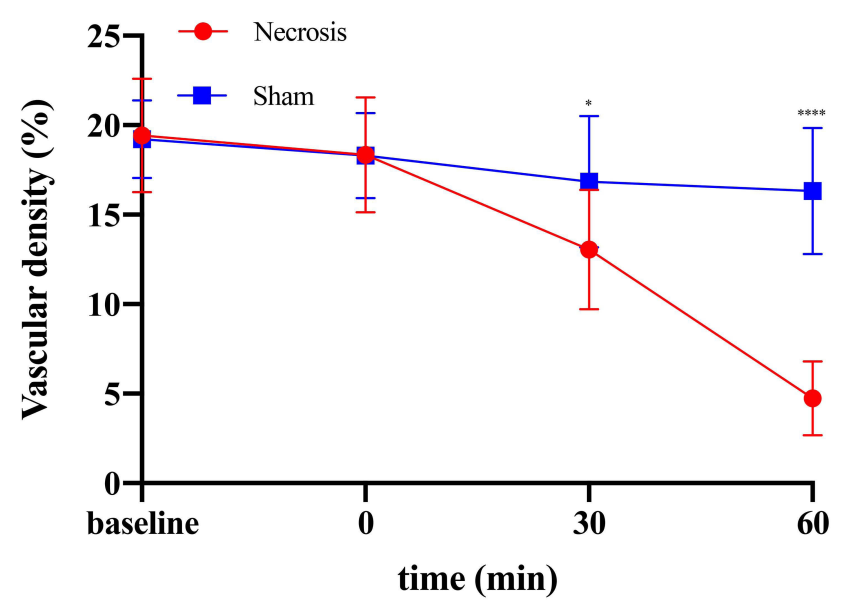

Figure 6 Quantitative analysis of vascular density in ROls detected by PAM between two groups. ${ }^{*} \mathrm{p}<0.05$, **** $\mathrm{p}<0.0001$.

demand. In recent years, PAM has attracted much attention in various basic and clinical studies based on its good performance in visualizing the microvessels. The technology was used to get structural and functional images of brain which is very important for cerebral vessel disease; for example, it has been performed to realize the aim of early diagnosis and treatment for stroke, Alzheimer's disease and ischemic neurodegeneration in animal models. ${ }^{29,30}$ In addition, as studies described previously, PAM can find more microvessels in thyroid/breast imaging, which is very important for early detection of tumors, compared with other modalities; and the data show that it holds great potential to distinguish between microvessels-nerves complexes and surrounding tissue intraoperatively in gynecological and urologic disorders, which is vital for surgeons to improve the clinical outcomes. ${ }^{31-33}$ Although PAM exhibits outstanding performance in mapping the microvessels, there are no studies involving the application of PAM in skin avulsion injuries. In our study, we developed a PAM system which takes advantage of high spatiotemporal resolution, deep penetration, multiple contrasts and high detection sensitivity. Our results showed that PA intensity and vascular density decreased significantly in the experimental group at $30 \mathrm{~min}$ and $60 \mathrm{~min}$ after surgery, which indicates this system could predict skin necrosis in skin avulsion as early as 30 min after injury. It would give more possibilities for surgeons to manage this injury, and further to better the clinical outcome.

\section{Conclusion}

Taken together, this study explicitly shows that the PAM image system can be used as an effective imaging technology tool for clinical application in skin avulsion injuries, mainly based on the early prediction of skin necrosis in a noninvasive and dynamic way. 


\section{Acknowledgments}

This study was supported by the Health Commission of Hubei Province Medical Leading Talent Project (Grant No. LJ20200405).

\section{Disclosure}

There are no financial or other conflicts of interest to disclose.

\section{References}

1. Rodewald M, Gundelach T, Degregorio N, Janni W, Hancke K. Management of "difficult" wounds. Eur J Pediatr Surg. 2013;23 (05):365-374. doi:10.1055/s-0033-1354588

2. Sakai G, Suzuki T, Hishikawa T, Shirai Y, Kurozumi T, Shindo M. Primary reattachment of avulsed skin flaps with negative pressure wound therapy in degloving injuries of the lower extremity. Injury. 2017;48(1):137-141. doi:10.1016/j.injury.2016.10.026

3. Jeng SF, Hsieh CH, Lin TS, Kuo YR, Wei FC. Classification and reconstruction options in foot plantar skin avulsion injuries: follow-up. Plast Reconstr Surg. 2003;112(1):220-221. doi:10.1097/ 01.PRS.0000066384.90361.5C

4. Arnez ZM, Khan U, Tyler MP. Classification of soft-tissue degloving in limb trauma. J Plast Reconstr Aesthet Surg. 2010;63 (11):1865-1869. doi:10.1016/j.bjps.2009.11.029

5. Boettcher-Haberzeth S, Schiestl C. Management of avulsion injuries. Eur J Pediatr Surg. 2013;23(05):359-364. doi:10.1055/s-0033-1353493

6. LaTulip S, Rao RR, Sielaff A, Theyyunni N, Burkhardt J. Ultrasound utility in the diagnosis of a morel-lavallée lesion. Case Rep Emerg Med. 2017;2017:3967587. doi:10.1155/2017/3967587

7. Bamba R, Malhotra G, Bueno RA, Thayer WP, Shack RB. Ring avulsion injuries: a systematic review. Hand (N Y). 2018;13 (1):15-22. doi:10.1177/1558944717692094

8. Kara A, Akin S, Ince C. Monitoring microcirculation in critical illness. Curr Opin Crit Care. 2016;22(5):444-452. doi:10.1097/ MCC. 0000000000000335

9. Khodaee M, Deu RS, Mathern S, Bravman JT. Morel-lavallée lesion in sports. Curr Sports Med Rep. 2016;15(6):417-422. doi:10.1249/ JSR.0000000000000306

10. Matsui A, Lee BT, Winer JH, Laurence RG, Frangioni JV. Predictive capability of near-infrared fluorescence angiography in submental perforator flap survival. Plast Reconstr Surg. 2010;126 (5):1518-1527. doi:10.1097/PRS.0b013e3181ef8ce7

11. Spain JA, Rheinboldt M, Parrish D, Rinker E. Morel-lavallée injuries: a multimodality approach to imaging characteristics. Acad Radiol. 2017;24(2):220-225. doi:10.1016/j.acra.2016.08.029

12. Abdelfattah U, Power HA, Song S, Min K, Suh HP, Hong JP. Algorithm for free perforator flap selection in lower extremity reconstruction based on 563 cases. Plast Reconstr Surg. 2019;144 (5):1202-1213. doi:10.1097/PRS.0000000000006167

13. Mücke T, Fichter AM, Schmidt LH, Mitchell DA, Wolff KD, Ritschl LM. Indocyanine green videoangiography-assisted prediction of flap necrosis in the rat epigastric flap using the flow $\left({ }^{\circledR}\right) 800$ tool. Microsurgery. 2017;37(3):235-242. doi:10.1002/micr.30072

14. Shinomiya R, Sunagawa T, Nakashima Y, et al. Monitoring vascular compromise using ultrasound after free tissue transfer. J Ultrasound Med. 2020;39(6):1213-1222. doi:10.1002/jum.15210
15. Ma H, Xiong K, Wu J, Ji X, Yang S. Noncontact photoacoustic angiography with an air-coupled ultrasonic transducer for evaluation of burn injury. Appl Phys Lett. 2019;114(13):133701. doi:10.1063/1.5088857

16. Qin W, Jin T, Guo H, Xi L. Large-field-of-view optical resolution photoacoustic microscopy. Opt Express. 2018;26(4):4271-4278. doi:10.1364/OE.26.004271

17. Hu S, Wang LV. Photoacoustic imaging and characterization of the microvasculature. J Biomed Opt. 2010;15(1):011101. doi:10.1117/ 1.3281673

18. Wang Y, Xing D, Zeng Y, Chen Q. Photoacoustic imaging with deconvolution algorithm. Phys Med Biol. 2004;49(14):3117-3124. doi:10.1088/0031-9155/49/14/006

19. Steinberg I, Huland DM, Vermesh O, Frostig HE, Tummers WS, Gambhir SS. Photoacoustic clinical imaging. Photoacoustics. 2019;14:77-98. doi:10.1016/j.pacs.2019.05.001

20. Junjie Y, Lihong V. Photoacoustic microscopy. Laser Photon Rev. 2013;7(5):758-778. doi:10.1002/lpor.201200060

21. American National Standards Institute. American National Standard for Safe Use of Lasers. Laser Institute of America; 2007.

22. Li Y, Hu X, Yi W, et al. NIR-II fluorescence imaging of skin avulsion and necrosis. Front Chem. 2019;7:696. doi:10.3389/ fchem.2019.00696

23. The National Pressure Ulcer Advisory Panel. Pressure ulcers prevalence, cost and risk assessment: consensus development conference statement. Decubitus. 1989;2(2):24-28.

24. Association AD. Diagnosis and classification of diabetes mellitus. Diabetes Care. 2010;33(Suppl 1):S62.

25. Chin MS, Chappell AG, Giatsidis G, et al. Hyperspectral imaging provides early prediction of random axial flap necrosis in a preclinical model. Plast Reconstr Surg. 2017;139(6):1285e-1290e. doi:10.1097/PRS.0000000000003352

26. Ural A, Bilgen F, Altıntaş Aykan D, Bahar AY, Bekerecioğlu M, Altıntaş ural D. Effects of dabigatran and fondaparinux on degloving injuries: an experimental study. Ulus Travma Acil Cerrahi Derg. 2020;26(3):343-350. doi:10.14744/tjtes.2020.26678

27. Bonilla-Yoon I, Masih S, Patel DB, et al. The Morel-Lavallée lesion: pathophysiology, clinical presentation, imaging features, and treatment options. Emerg Radiol. 2014;21(1):35-43. doi:10.1007/s10140013-1151-7

28. Goodman BS, Smith MT, Mallempati S, Nuthakki P. A comparison of ultrasound and magnetic resonance imaging findings of a MorelLavallée lesion of the knee. $P M \& R$. 2013;5(1):70-73. doi:10.1016/j. pmrj.2012.08.001

29. Cao R, Li J, Kharel Y, et al. Photoacoustic microscopy reveals the hemodynamic basis of sphingosine 1-phosphate-induced neuroprotection against ischemic stroke. Theranostics. 2018;8(22):6111-6120. doi: $10.7150 /$ thno. 29435

30. Cao R, Li J, Zhang C, Zuo Z, Hu S. Photoacoustic microscopy of obesity-induced cerebrovascular alterations. Neuroimage. 2019;188:369-379. doi:10.1016/j.neuroimage.2018.12.027

31. Yang F, Wang Z, Zhang W, et al. Wide-field monitoring and real-time local recording of microvascular networks on small animals with a dual-raster-scanned photoacoustic microscope. J Biophoton. 2020;13 (6):e202000022.

32. Yang S, Da X, Lao Y, et al. Noninvasive monitoring of traumatic brain injury and post-traumatic rehabilitation with laser-induced photoacoustic imaging. Appl Phys Lett. 2007;90(24):629. doi:10.1063/1.2749185

33. Wu Z, Duan F, Zhang J, Li S, Ma H, Nie L. In vivo dual-scale photoacoustic surveillance and assessment of burn healing. Biomed Opt Express. 2019;10(7):3425-3433. doi:10.1364/BOE.10.003425 


\section{Publish your work in this journal}

Clinical, Cosmetic and Investigational Dermatology is an international, peer-reviewed, open access, online journal that focuses on the latest clinical and experimental research in all aspects of skin disease and cosmetic interventions. This journal is indexed on CAS.

The manuscript management system is completely online and includes a very quick and fair peer-review system, which is all easy to use. Visit http://www.dovepress.com/testimonials.php to read real quotes from published authors.

Submit your manuscript here: https://www.dovepress.com/clinical-cosmetic-and-investigational-dermatology-journal 